This is an electronic reprint of the original article. This reprint may differ from the original in pagination and typographic detail.

Author(s): Ramasubramaniam, A.; Koskinen, Pekka; Kit, Oleg; Shenoy, V.B.

Title: $\quad$ Edge-stress -induced spontaneous twisting of graphene nanoribbons

Year: $\quad 2012$

Version:

Please cite the original version:

Ramasubramaniam, A., Koskinen, P., Kit, O., \& Shenoy, V.B. (2012). Edge-stress induced spontaneous twisting of graphene nanoribbons. Journal of Applied Physics, 111(5). https://doi.org/10.1063/1.3689814

All material supplied via JYX is protected by copyright and other intellectual property rights, and duplication or sale of all or part of any of the repository collections is not permitted, except that material may be duplicated by you for your research use or educational purposes in electronic or print form. You must obtain permission for any other use. Electronic or print copies may not be offered, whether for sale or otherwise to anyone who is not an authorised user. 


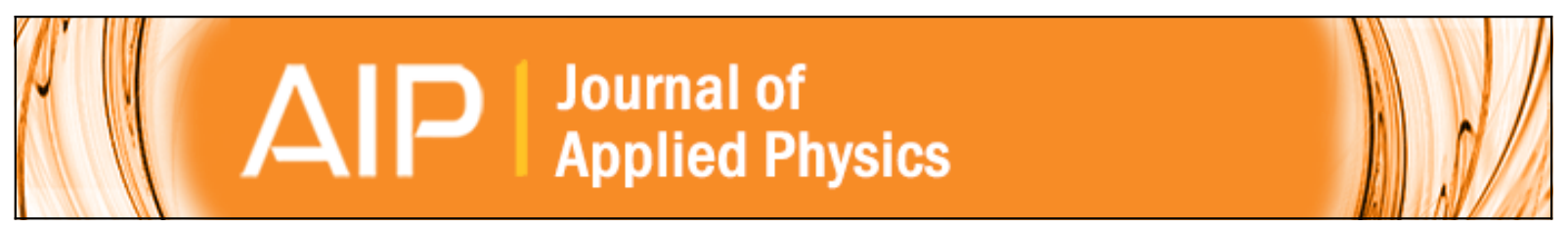

\section{Edge-stress-induced spontaneous twisting of graphene nanoribbons}

Ashwin Ramasubramaniam, Pekka Koskinen, Oleg O. Kit, and Vivek B. Shenoy

Citation: Journal of Applied Physics 111, 054302 (2012); doi: 10.1063/1.3689814

View online: http://dx.doi.org/10.1063/1.3689814

View Table of Contents: http://scitation.aip.org/content/aip/journal/jap/111/5?ver=pdfcov

Published by the AIP Publishing

\section{Articles you may be interested in}

Band gap engineering in finite elongated graphene nanoribbon heterojunctions: Tight-binding model AIP Advances 5, 087121 (2015); 10.1063/1.4928450

Influence of zigzag edges on the Ruderman-Kittel-Kasuya-Yosida interaction between magnetic impurities in graphene nanoribbons

J. Appl. Phys. 116, 194309 (2014); 10.1063/1.4902146

Excitonic effects of E11, E22, and E33 in armchair-edged graphene nanoribbons

J. Appl. Phys. 115, 103701 (2014); 10.1063/1.4867937

Chiral graphene nanoribbons: Objective molecular dynamics simulations and phase-transition modeling

J. Chem. Phys. 137, 234702 (2012); 10.1063/1.4770002

Electromechanics of twisted graphene nanoribbons

Appl. Phys. Lett. 99, 013105 (2011); 10.1063/1.3607956

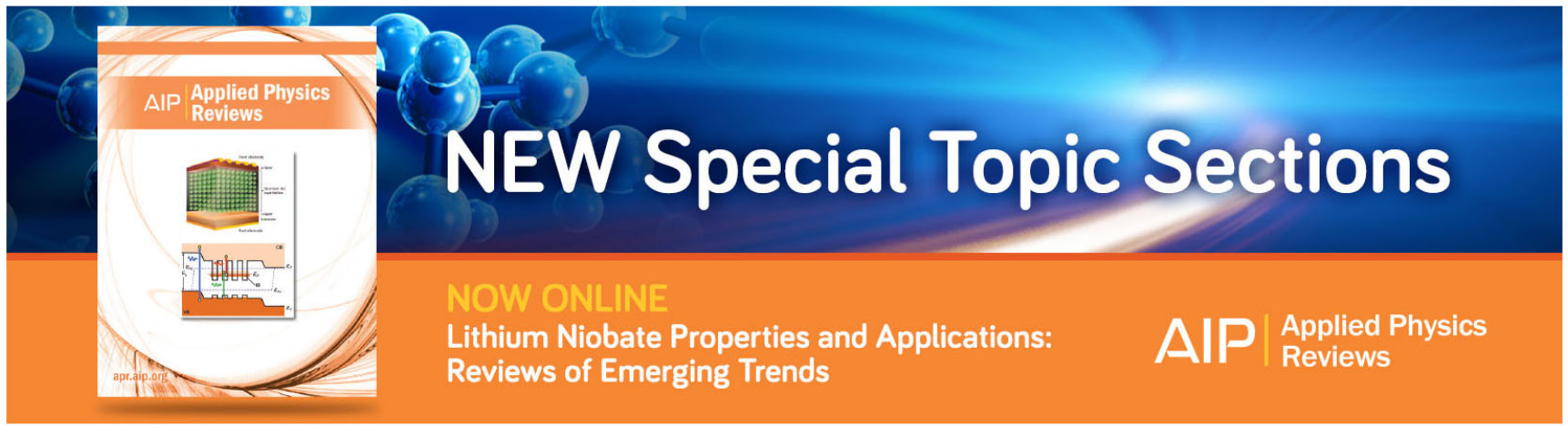




\title{
Edge-stress-induced spontaneous twisting of graphene nanoribbons
}

\author{
Ashwin Ramasubramaniam, ${ }^{1, a)}$ Pekka Koskinen, ${ }^{2, b)}$ Oleg O. Kit, ${ }^{2}$ and Vivek B. Shenoy ${ }^{3, c)}$ \\ ${ }^{1}$ Department of Mechanical and Industrial Engineering, University of Massachusetts Amherst, Amherst, \\ Massachusetts 01003, USA \\ ${ }^{2}$ NanoScience Center, Department of Physics, University of Jyväskylä, 40014 Jyväskylä, Finland \\ ${ }^{3}$ School of Engineering, Brown University, Providence, Rhode Island 02912, USA
}

(Received 15 July 2011; accepted 2 February 2012; published online 2 March 2012)

\begin{abstract}
We present a continuum model for spontaneous twisting of graphene nanoribbons driven by compressive edge stresses. Based on a geometrically nonlinear theory of plates, we identify scaling laws for the dependence of twist angles on ribbon width. Strikingly, we find the existence of a critical width below which a ribbon will not undergo spontaneous twisting, preferring an in-plane stretching mode instead. The model predictions are shown to be in excellent qualitative and quantitative agreement with density-functional tight-binding simulations. More generally, our model provides a unifying picture of twisting in graphene nanoribbons with different edge orientations and chemical functionalizations that have been reported recently in the literature. (C) 2012 American Institute of Physics. [http://dx.doi.org/10.1063/1.3689814]
\end{abstract}

\section{INTRODUCTION}

Graphene-a two-dimensional atomically thin layer of $s p^{2}$ bonded carbon atoms - has been the focus of extensive research in recent years in part due to its extraordinary electronic properties, which make it a promising candidate for next-generation electronic devices. ${ }^{1,2}$ While large-area graphene does not present a usable bandgap for digital electronics, quantum confinement of charge carriers in $1 \mathrm{D}$ graphene nanoribbons (GNRs) or 0 D quantum dots provides reasonable band gaps for applications. For $1 \mathrm{D}$ nanoribbons, in particular, theoretical and experimental studies demonstrate that band gaps scale inversely with ribbon width, ${ }^{3-6}$ providing an avenue for band-gap engineering in graphene.

While the width of a GNR establishes the essential inverse scaling relationship of band gaps, the nature of the ribbon edge is critical in establishing the quantitative details. Zigzag GNRs (ZGNRs) display small band gaps arising from a staggered sublattice potential due to spin-ordered states at the edges. ${ }^{3}$ Intermediate orientations (chiral GNRs) also show the presence of magnetic edge states. ${ }^{7}$ Armchair GNRs (AGNRs) do not display such spin-ordered edge-states but nevertheless understanding the trends and scaling in their band gaps requires the incorporation of changes in $\mathrm{C}-\mathrm{C}$ bond lengths along the edges. ${ }^{3}$ GNR edges have also been shown to undergo reconstructions (5-6 and 5-7 reconstructed edges), which alter the electronic properties of the ribbon. ${ }^{8-10}$ Additionally, edges are more chemically reactive due to the presence of dangling bonds and chemical functionalization/ contamination can further alter the edge electronic properties and thence the overall behavior of the GNR. ${ }^{11-15}$

From a macroscopic thermodynamics perspective, the edge of a GNR is associated with a scalar edge stress in analogy with a tensorial surface stress for 3 D crystals. ${ }^{16,17}$

\footnotetext{
${ }^{\text {a)} E l e c t r o n i c ~ m a i l: ~ a s h w i n @ e n g i n . u m a s s . e d u . ~}$

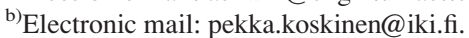

c)Electronic mail: vivek_shenoy@brown.edu.
}

The underlying physical cause for this edge stress is the change in the bonding environment of edge atoms (which are missing neighbors) from their ideal bulk state. Computational studies show that the edges of pristine ZGNRs and AGNRs are both under a state of compressive edge stress, whereas 5-6 and 5-7 reconstructed edges display tensile edge stresses. ${ }^{17-20}$ The edge stress is sensitive to chemical functionalization as this can significantly alter the local environment of edge atoms. Thus, hydrogen termination of edges can lead to a precipitous drop in the compressive edge stress for AGNRs and ZGNRs. ${ }^{17-19}$ Fluorination, on the other hand, appears to lead to enhanced compressive edge stresses due to ionic repulsion between fluorine atoms. ${ }^{24}$ In general, edge stresses lead to an excess elastic edge energy, which can typically be relieved efficiently by non-planar deformations, such as buckling, warping, scrolling, and twisting. ${ }^{16,20-23}$ Of course, relieving the edge energy through such deformations comes at a cost since some energy must now be stored in the "bulk" of the GNR. Therefore, the preferred deformation modes for a GNR are set by this competition between surface and bulk energy and as such are dependent upon its physical dimensions.

Among several possible deformation modes, GNRs are susceptible to a particularly interesting edge-stress driven twisting mode. ${ }^{24,25}$ These twisting modes couple strongly to the electronic structure of the GNR and can effectively tune its electronic bandgap over a wide range $;{ }^{24,26}$ this electromechanical response could lead to applications in switches or sensors. While previous studies ${ }^{24,26}$ have considered the role of twisting on electronic structure quite thoroughly, there is as yet no simple theory that elucidates the mechanics of GNR twisting. Bets and Yakobson ${ }^{25}$ have partially addressed this issue through empirical potential calculations and shown the existence of a bifurcation from edge-stress driven twisting modes to localized edge-rippling modes with increasing ribbon width. In this paper, we develop a comprehensive continuum model for edge-stress driven buckling of GNRs. The key results of our model are as follows. First, 
compressive edge stresses induce energy minima at non-zero twist angles, which provide the driving force for spontaneous twisting (as seen in unterminated and F-terminated GNRs). Second, there exists a critical width below which GNRs will preferentially undergo in-plane stretching rather than (out-of-plane) twisting. Intuitively, these ultra-thin GNRs are "all edge" and the energy cost for planar stretching of the entire structure is lesser than for out-of-plane twisting. Third, with increasing width $w$, the twist of a GNR scales as $1 / w^{3 / 2}$ (rather than $1 / w$ as deduced computationally in Ref. 25). Our theoretical predictions are shown to be in good qualitative and reasonable quantitative agreement with tight-binding simulations of twisting of unterminated AGNRs. While we restrict attention in this work to unterminated AGNRs, our model is more generally applicable to other situations where GNRs experience compressive edge stresses (e.g., unterminated ZGNRs, F-terminated GNRs, etc.). Since the electronic properties of GNRs can be modulated by twisting, our work provides a route for engineering electromechanical coupling in GNRs by controlling edge structure and/or chemical functionalization of edges.

\section{RESULTS AND DISCUSSION}

\section{A. Analytical model for twisting}

The graphene nanoribbon is treated as a 2 D elastic strip of length $\lambda_{0}$ and width $w$, with $w / \lambda_{0} \ll 1$ (see Fig. 1). The strip is taken to be periodic along its length. First, suppose the ribbon is twisted into a helical strip while keeping the translational periodic length $\lambda_{0}$ constant. With reference to the kinematics of twisting illustrated in Fig. 2, it is readily shown that a material fiber parallel to the axis will experience an extensional strain $\epsilon_{y y}=x^{2} \Theta_{0}^{2} / 2$ in the limit $w / \lambda_{0} \ll$ 1 , where $\Theta_{0}=2 \pi / \lambda_{0}$ is the twist. In the absence of axial constraints on the ribbon, it is clear that the ribbon must contract to some optimal length $\lambda<\lambda_{0}$ in order to accommodate the non-uniform stretching of axial fibers. Thus, the actual axial strain in the ribbon is given by

$$
\epsilon_{y y}(x)=\epsilon_{0}+\frac{1}{2} \Theta^{2} x^{2} \equiv \epsilon_{0}+\frac{2 \pi^{2} x^{2}}{\lambda^{2}},
$$

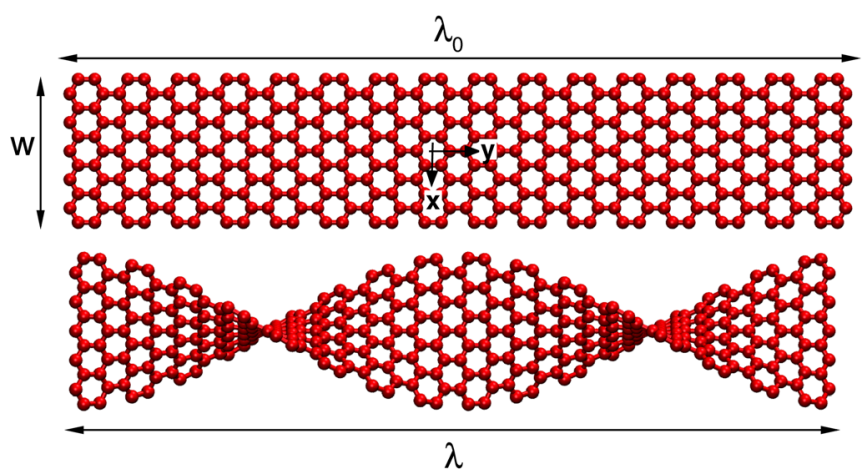

FIG. 1. (Color online) Schematic of a graphene nanoribbon of width $w$ and translational periodic length $\lambda$ undergoing twisting. Upon twisting, and in the absence of axial constraints, the axial length of the ribbon decreases to an optimal value $\lambda<\lambda_{0}$

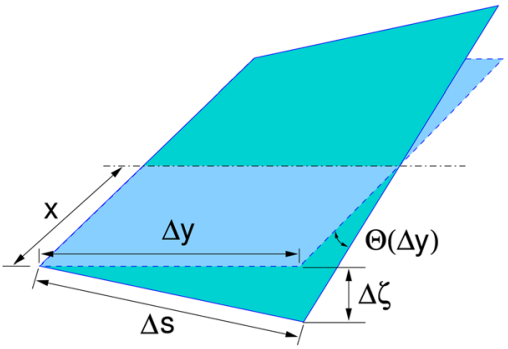

FIG. 2. (Color online) Strip of width $2 x$ and length $\Delta y$ undergoing a twist through an angle $\Theta \times \Delta y$. If the length of the ribbon is held fixed during twisting, the axial strain in the fiber at distance $x$ from the ribbon axis is $\epsilon_{y y}=\lim _{\Delta y \rightarrow 0}[(\Delta s / \Delta y)-1] \approx x^{2} \Theta^{2} / 2$ for $x / \lambda \ll 1$. The mean curvature of the strip is zero while the Gaussian curvature is $\Theta^{2}$.

where $\Theta=2 \pi / \lambda$ is now the twist with respect to the optimized length $\lambda$, and $\epsilon_{0}=\left(\lambda / \lambda_{0}-1\right)<0$, which we will calculate explicitly later, is the (compressive) strain experienced by an axial fiber situated along the symmetry axis of the ribbon $(x=0)$. The energy cost per unit length associated with this in-plane stretching of the sheet is given by

$$
\begin{aligned}
E_{\text {stretch }} & =\frac{1}{2} \int_{-w / 2}^{w / 2} Y \epsilon_{y y}^{2} d x \\
& =\frac{Y w}{2}\left(\epsilon_{0}^{2}+\frac{1}{12} w^{2} \epsilon_{0} \Theta^{2}+\frac{w^{4} \Theta^{4}}{320}\right),
\end{aligned}
$$

where $Y$ is the 2 D Young's modulus of graphene.

Next, with reference to the kinematics illustrated in Fig. 2, it is straightforward to show that the second derivatives of the out of plane displacement $\zeta(x, y)$ are $\partial^{2} \zeta / \partial x^{2}$ $=\partial^{2} \zeta / \partial y^{2}=0$ and $\partial^{2} \zeta / \partial x \partial y=\Theta$, from which it follows that the bending energy cost per unit length ${ }^{27}$ is

$$
\begin{aligned}
E_{\text {bend }}= & \frac{1}{2} B \int_{-w / 2}^{w / 2}\left[\left(\frac{\partial^{2} \zeta}{\partial x^{2}}+\frac{\partial^{2} \zeta}{\partial y^{2}}\right)^{2}\right. \\
& \left.+2(1-v)\left\{\left(\frac{\partial^{2} \zeta}{\partial x \partial y}\right)^{2}-\frac{\partial^{2} \zeta}{\partial x^{2}} \frac{\partial^{2} \zeta}{\partial y^{2}}\right\}\right] d x, \\
= & B w(1-v) \Theta^{2},
\end{aligned}
$$

$B$ and $\nu$ being the $2 \mathrm{D}$ bending modulus and Poisson's ratio of the sheet, respectively. Unlike a plate, $Y$ and $B$ are independent for a $2 \mathrm{D}$ sheet, although they can be artificially related to each other if need be (e.g., for finite element simulations) by assuming an effective thickness $h$ for the sheet such that the standard plate-theory relation $B=Y h^{3}$ / $12\left(1-\nu^{2}\right)$ is satisfied. ${ }^{16,17}$

The final contribution to the energy stored in the twisted ribbon comes from (a) the presence of an edge stress and (b) stretching and bending energies for the edge, whose elastic properties can differ from those in the bulk due to the difference in the bonding environment. ${ }^{16,17}$ The energy per unit length arising from these two factors is

$$
E_{\text {edge }}=2\left[\tau \epsilon_{y y}+\frac{1}{2} Y_{e} \epsilon_{y y}+\frac{1}{2} B_{e} \Theta^{2}\right]_{x=w / 2},
$$

where $Y_{e}$ is the $1 \mathrm{D}$ Young's modulus of the edge, $B_{e}$ is an edge bending modulus, and $\tau$ is the edge stress; the prefactor 
of 2 accounts for both edges. As noted in our previous work, ${ }^{16}$ the edge is essentially treated as a $1 \mathrm{D}$ fiber that is either stretched or compressed (effectively generating an edge stress) and "glued" to a bulk with different elastic properties. In principle, such a $1 \mathrm{D}$ fiber can display a resistance to both stretching and bending.

The total energy per unit length of the twisted ribbon is given by

$$
\begin{aligned}
E\left(\epsilon_{0}, \Theta ; w\right)= & E_{0}+E_{\text {stretch }}+E_{\text {bend }}+E_{\text {edge }} \\
= & E_{0}+\frac{Y w}{2}\left(\epsilon_{0}^{2}+\frac{1}{12} w^{2} \epsilon_{0} \Theta^{2}+\frac{w^{4} \Theta^{4}}{320}\right) \\
& +\Theta^{2} B w(1-v) \\
& +2\left[\tau \epsilon_{y y}+\frac{1}{2} Y_{e} \epsilon_{y y}^{2}+\frac{1}{2} B_{e} \Theta^{2}\right]_{x=w / 2},
\end{aligned}
$$

where $E_{0}$ is the energy per unit length of the ribbon in its undeformed state. The optimal strain $\epsilon_{0}^{*}$ for a given twist $\Theta$ is readily obtained as

$$
\epsilon_{0}^{*}=-\frac{w^{2} \Theta^{2}}{24} \frac{6 Y_{e}+Y w}{2 Y_{e}+Y w}-\frac{2 \tau}{2 Y_{e}+Y w} .
$$

An additional optimization of the energy with respect to $\Theta$ gives us the following solutions for the optimal twist

$$
\begin{aligned}
& \Theta^{*}=0 \text { and } \Theta^{*} \\
& = \pm 2 \sqrt{30} \frac{\sqrt{-6 B_{e}\left(2 Y_{e}+Y w\right)-6 B w\left(2 Y_{e}+Y w\right)(1-v)-Y w^{3} \tau}}{\sqrt{Y w^{5}\left(12 Y_{e}+Y w\right)}}
\end{aligned}
$$

from which it is apparent that the edge stress $\tau$ must be negative (compressive) for non-zero values of $\Theta *$ to exist $\left(B, B_{e}\right.$, $Y_{e}, Y, w, \nu$ being positive). If such non-zero solutions exist, it is readily shown that these correspond to the two minima of a double-well potential symmetric about a local maximum at $\Theta=0$. Thus, ribbons with unterminated zigzag/armchair edges, which experience compressive edge stresses, ${ }^{16-18}$ can be expected to twist spontaneously, whereas ribbons with 5-6 and 5-7 reconstructed edges, ${ }^{17,19}$ which experience tensile edge stresses will not twist spontaneously but simply stretch in plane (within the kinematics allowed for here). Furthermore, Eq. (7) also shows that there is a critical width $w^{*}$ below which real-valued solutions for $\Theta *$ will cease to exist even for negative $\tau$ [i.e., when $Y w^{3}|\tau|<6 B_{e}\left(2 Y_{e}+Y w\right)$ $\left.+6 B w\left(2 Y_{e}+Y w\right)(1-\nu)\right]$. In other words, a compressive edge stress is in of itself insufficient to cause spontaneous twisting; it is necessary that the ribbon width also exceed a critical value $w^{*}$ for this to occur. Below this critical width $w^{*}$, ribbons will cease to twist spontaneously and undergo only in-plane stretching. Physically, this result reflects the interplay between bulk and edge energy. For ribbon widths lesser than $w^{*}$, the tendency for the edge to spontaneously elongate can be accommodated by stretching the ribbon bulk without an excessive energy cost. Beyond this critical width, simply stretching the bulk carries too high an energy penalty; twisting, which is another mode of accommodating edge-stretching with lesser in-plane stretching, becomes energetically favorable. These features will be illustrated later in Sec. II B in conjunction with actual computational studies. Finally, we also see that as the ribbon starts to become very wide (sheet-like) the optimal twist decreases to leading order as $1 / w^{3 / 2}$.

\section{B. Comparison of theory and simulations of ribbon twisting}

To demonstrate the validity of our analytical model we now compare the analytical results presented above with tight-binding simulations of twisting of graphene nanoribbons that use the revised Bloch approach developed by Koskinen and $\mathrm{Kit}^{28}$ (see Methods). For concreteness, we focus on unterminated AGNRs, which have been shown to spontaneously undergo edge-stress induced twisting in previous work. ${ }^{28}$ The same behavior should also be observed in zigzag graphene nanoribbons or, more generally, for any edge orientation that is associated with compressive edge stresses.

The material parameters for the analytical model, as determined from tight-binding simulations, are $B=1.61 \mathrm{eV}$, $Y=25.47 \mathrm{eV} / \AA^{2}, \tau=-1.7 \mathrm{eV} / \AA$, and $\nu=0.28$. The edge moduli $Y_{e}$ and $B_{e}$ are treated as fitting parameters to the tightbinding data for AGNRs of different widths, displayed by symbols in Fig. 3. A two-parameter fit to these data via Eq. (5) gives values of $Y_{e}=18.69 \mathrm{eV} / \AA$ and $B_{e}=15.01 \mathrm{eV} \cdot \AA$. The strain energy relieved by twisting $\Delta E\left[\epsilon_{0}^{*}(\Theta), \Theta ; w\right]$ $=E\left[\epsilon_{0}^{*}(\Theta), \Theta ; w\right]-E\left[\epsilon_{0}^{*}(0), 0 ; w\right]$ can now be obtained from Eq. (5) as a function of twist $\Theta$ for various ribbon widths, which we display in Fig. 3. The presence of a minimum in the energy curve at non-zero values of $\Theta^{*}$, indicating the tendency to twist spontaneously, is apparent for the three

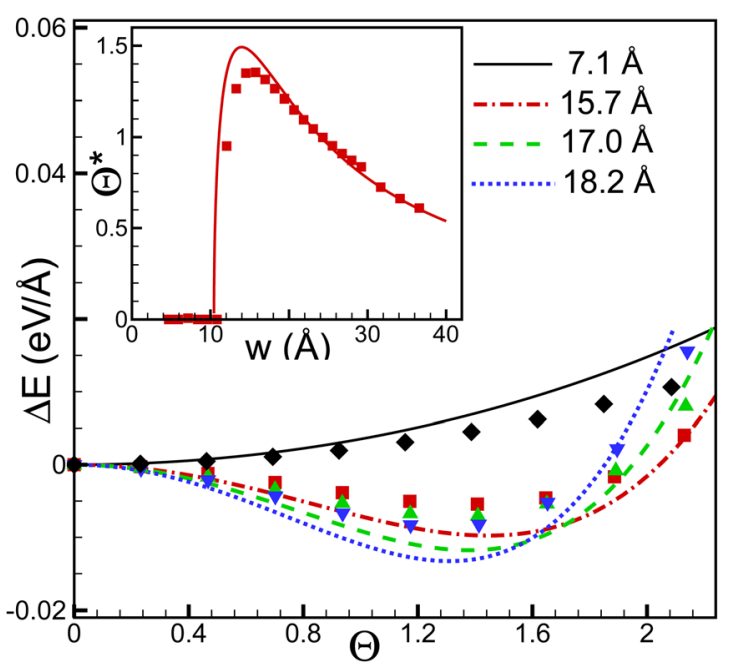

FIG. 3. (Color online) Change in energy per unit length $\Delta E=E\left[\epsilon_{0}^{*}(\Theta), \Theta ; w\right]$ $-E\left[\epsilon_{0}^{*}(0), 0 ; w\right]$ of the nanoribbon as a function of twist $\Theta$ (in $\operatorname{deg} / \AA$ ). A minimum away from $\Theta=0$, when present, indicates an energetic preference for spontaneous twisting of the ribbon driven by edge stresses. The inset shows the optimum twist $\Theta^{*}$ (minimum in the $\Delta E$ vs. $\Theta$ curve) as a function of ribbon width. As seen, the optimal twist is maximum for ribbons in the range of $14-16 \AA$, reflecting a compromise between energy stored due to bending and stretching. It is also apparent that the thinnest ribbon ( $w=7.1 \AA$ ) is below the critical width for spontaneous twisting and has an energy minimum at $\theta=0$. The symbols are data from tight-binding simulations while like-colored lines are the corresponding predictions from elasticity theory. 
widest ribbons. With increasing ribbon width the energy minima become shallower and shift toward smaller values of $\Theta$. The dependence of the optimal twist $\Theta *$ on ribbon width is shown more clearly in the inset of Fig. 3. It is readily observed that the optimal twist goes to zero below a critical width $w^{*}$ as alluded to before in Sec. II A. The analytical estimate for this width is $10.5 \AA$ while the computational estimate is $10.8 \AA$. The thinnest ribbon $(w=7.1 \AA)$ considered here falls below this critical width and thus does not show a tendency for spontaneous twisting; this is clearly evidenced from the energy versus twist data for the ribbon, which has a minimum at $\Theta=0$. The inset of Fig. 3 also shows that there is an optimal ribbon width (set by material parameters and edge orientation) which gives the maximum possible spontaneous twist for a GNR; this width is estimated to be about $14.0 \AA$ and $15.7 \AA$ from the analytical and computational data, respectively. Finally, the asymptotic decay of the twist with increasing width is also clearly observed in these data. Overall, the analytical model clearly captures all the qualitative features of the simulations, with reasonable quantitative agreement as well. Most strikingly, the analytical model clearly captures the existence of a critical width for spontaneous twisting to within $4 \%$ accuracy (analytical model $\sim 10.4 \AA$, computed $\sim 10.8 \AA$ ). Figure 4 shows the variation in the optimal axial strain $\epsilon_{0}^{*}(\Theta)$ as a function of twist $\Theta$ (Eq. (6)). The tight-binding data and analytical estimates are in fair overall agreement. The quantitative discrepancies at small twists likely arise from the a combination of the inevitable residual forces acting on the unit cell at the end of geometry optimization as well as other details at the atomic scale that are not described by the continuum model; deviations at large twists are to be expected, of course, due to nonlinearities in the constitutive response of the material (strains are a few percent). Note also that the widths $w$ in continuum and atomistic pictures cannot be compared directly; the connection is inherently ambiguous. Previous simulations by Zhang and Dumitrică ${ }^{26}$ have also reported similar results of axial contraction of graphene nanoribbons due to twisting.

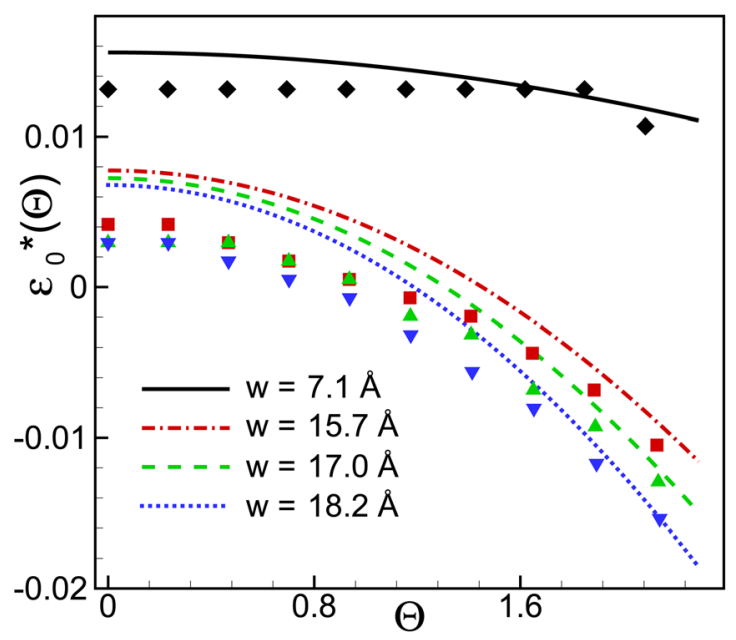

FIG. 4. (Color online) Optimal axial strain $\epsilon_{0}^{*}$ of the nanoribbon as a function of twist angle $\Theta$ (in deg/ $\AA$ ). Larger angles of twist $\Theta$ lead to larger differential stretching of axial fibers leading to smaller optimal axial lengths (more negative $\epsilon_{0}^{*}$ ). The symbols (matched by color with the lines) are data from tight-binding simulations while the lines are theoretical predictions.
In concluding this discussion, we note that the analytical model developed here can be applied without any essential modification (except for appropriate edge stresses and edge moduli) to twisting of AGNRs with fluorinated edges studied recently by Gunlycke et $a{ }^{24}$ In that work, the authors showed the tendency for F-terminated AGNRs to twist spontaneously due to an "ionic repulsion between neighboring $\mathrm{F}$ atoms," which in essence amounts to a compressive edge stress within the continuum picture. They also showed that H-terminated AGNRs, in contrast, do not show this tendency to twist spontaneously, displaying only a minimum at zero twist angle. This result is also readily explained within the continuum picture by recognizing that $\mathrm{H}$-termination of AGNRs causes the edge stress to become extremely small ${ }^{17-19}$ thereby causing the optimal twist angle (Eq. (7)) to go to zero.

\section{CONCLUDING REMARKS}

In summary, we have presented a continuum model for edge-stress induced spontaneous twisting of GNRs and validated this against tight-binding simulations. The driving force for twisting arises from compressive edge stresses, which are a manifestation of the tendency for the edge to expand. We have shown that there is a critical ribbon width below which in-plane stretching of the entire ribbon is favored over out-of-plane twisting. At the other extreme, the optimal twist scales as $1 / w^{3 / 2}$ for large ribbon widths, essentially giving way to more favorable deformation modes, such as localized edge-rippling. While our numerical validation was performed in the context of twisting of unterminated AGNRs, the continuum model is of much broader applicability and can be used to predict optimal twists and axial strains for any edge orientation or chemical functionalization as long as the edge stress is compressive. In essence, all that is needed from an atomic scale calculation (quantum mechanical or otherwise) are the elastic moduli and the edge stress. Since the electronic properties of GNRs can be controlled by twisting, our work provides a route for engineering electromechanical coupling in GNRs by controlling edge structure and/or chemical functionalization of edges. This electromechanical coupling in GNRs might be exploited in graphenebased switches or sensors in the future.

\section{A. Methods}

We simulated the ribbons using the spin-unpolarized density-functional tight-binding (DFTB) method, which is an established and computationally efficient method able to capture the elastic properties of honeycomb carbon with fair accuracy. ${ }^{29,30}$ Efficient simulation of the twisting itself was enabled by revised periodic boundary conditions (RPBC), where the conventional translational symmetry had been replaced by more general symmetry operations. ${ }^{28}$ Here we used the minimal RPBC simulation cell compatible with symmetry (short piece of the ribbon's other half), accompanied by two chiral symmetry operations, one counting for the ribbon's infinite extent, one for the ribbon's other half; these two chiral operations are described in more detail in 
Ref. 28. In particular, RPBC enables us to change the twist continuously.

Simulations were done by performing a full structural optimization (maximum force criterion $f_{\max } \leq 10^{-2} \mathrm{eV} / \AA$ ) with fixed simulation cell parameters (axial length and twist); there were no constraints within the cell. The symmetry operation-related $\kappa$-points were sampled with a regular $20 \times 2$-point mesh. Young's modulus and Poisson's ratio were determined by straining graphene (two-atom unit cell and conventional $\mathrm{PBC}$ with $20 \times 20 \mathbf{k}$-point mesh) in one direction while minimizing the energy with respect to the strain in the perpendicular direction. We conclude by remarking that the sole approximation in our atomistic twisting simulations is the DFTB method itself.

\section{ACKNOWLEDGMENTS}

A.R. gratefully acknowledges new faculty research funding from the University of Massachusetts Amherst. P.K. acknowledges the Academy of Finland and O.O.K. the National Graduate School of Material Physics (NGSMP) for funding. V.B.S. gratefully acknowledges support from the Army Research Office (Contract No. W911NF-11-1-0171).

${ }^{1}$ A. K. Geim and K. Novoselov, Nature Mater. 6, 183 (2007).

${ }^{2}$ A. H. C. Neto, F. Guinea, N. M. R. Peres, K. S. Novoselov, and A. K. Geim, Rev. Mod. Phys. 81, 109 (2009).

${ }^{3}$ Y.-W. Son, M. L. Cohen, and S. G. Louie, Phys. Rev. Lett. 97, 216803 (2006).

${ }^{4}$ Y. Han, B. Özyilmaz, Y. Zhang, and P. Kim, Phys. Rev. Lett. 98, 206805 (2007).

${ }^{5}$ Z. Chen, Y.-M. Lin, M. J. Rooks, and P. Avouris, Physica E 40, 228 (2007).
${ }^{6}$ X. Li, X. Wang, L. Zhang, S. Lee, and H. Dai, Science 319, 1229 (2008).

${ }^{7}$ O. V. Yazyev, R. B. Capaz, and S. G. Louie, Phys. Rev. B 84, 115406 (2011).

${ }^{8}$ P. Koskinen, S. Malola, and H. Hakkinen, Phys. Rev. Lett. 101, 115502 (2008).

${ }^{9}$ C. O. Girit, J. C. Meyer, R. Erni, M. D. Rossell, C. Kisielowski, L. Yang, C. H. Park, M. F. Crommie, M. L. Cohen, and S. G. Louie, Science 323, 1705 (2009).

${ }^{10}$ P. Koskinen, S. Malola, and H. Hakkinen, Phys. Rev. B 80, 073401 (2009).

${ }^{11}$ F. Cervantes-Sodi, G. Csányi, S. Pisanec, and A. C. Ferrari, Phys. Rev. B 77, 165427 (2008).

${ }^{12}$ D. Gunlycke, J. Li, J. W. Mintmire, and C. T. White, Appl. Phys. Lett. 91, 112108 (2007).

${ }^{13}$ O. Hod, V. Barone, J. E. Peralta, and G. E. Scuseria, Nano Lett. 7, 2295 (2007).

${ }^{14}$ G. Lee, and K. Cho, Phys. Rev. B 79, 165440 (2009).

${ }^{15}$ A. Ramasubramaniam, Phys. Rev. B 81, 245413 (2010).

${ }^{16}$ V. B. Shenoy, C. D. Reddy, A. Ramasubramaniam, and Y. W. Zhang, Phys. Rev. Lett. 101, 245501 (2008).

${ }^{17}$ C. D. Reddy, A. Ramasubramaniam, V. B. Shenoy, and Y. W. Zhang, Appl. Phys. Lett. 94, 101904 (2009).

${ }^{18}$ S. Jun, Phys. Rev. B 78, 073405 (2008).

${ }^{19}$ B. Huang, M. Liu, N. H. Su, J. Wu, W. H. Duan, B. L. Gu, and F. Liu, Phys. Rev. Lett. 102, 166404 (2009).

${ }^{20}$ V. B. Shenoy, C. D. Reddy, Y.-W. Zhang, ACS Nano 4, 4840 (2011).

${ }^{21}$ X. Xu and M. J. Buehler, ACS Nano 4, 3869 (2012).

${ }^{22}$ Q. Lu, Phys. Rev. B 81, 155410 (2012).

${ }^{23}$ Q. Lu, W. Gao, and R. Huang, Modelling Simul. Mater. Sci. Eng. 19, 054006 (2011).

${ }^{24}$ D. Gunlycke, J. Li, J. W. Mintmire, C. T. White, Nano Lett. 10, 3638 (2010).

${ }^{25}$ K. Bets and B. I. Yakobson, Nano Res. 2, 78 (2009).

${ }^{26}$ D. B. Zhang and, T. Dumitrică, Small 7, 1023 (2011).

${ }^{27}$ L. D. Landau and E. M. Lifshitz, Theory of Elasticity, 3rd ed. (Oxford, New York, 1986).

${ }^{28}$ P. Koskinen and O. O. Kit, Phys. Rev. Lett. 105, 106401 (2010).

${ }^{29}$ P. Koskinen and V. Mäkinen, Comput. Mater. Sci. 47, 237 (2009).

${ }^{30}$ D. Porezag, Th. Frauenheim,. Th. Köhler, G. Seifert, and R. Kaschner, Phys. Rev. B 51, 12947 (1995). 